\title{
Marx, éthicien anarchiste ?
}

Maximilien Rubel, Karl Marx. Essai de biographie intellectuelle, Paris :

Klincksieck, coll. « Critique de la politique », 2016 (464 p.)

Jean Quétier

\section{(2) OpenEdition}

\section{Journals}

Édition électronique

URL : https://journals.openedition.org/cps/302

DOI : $10.4000 /$ cps.302

ISSN : 2648-6334

Éditeur

Presses universitaires de Strasbourg

\section{Édition imprimée}

Date de publication : 9 mai 2017

Pagination : 161-165

ISBN : 978-2-86820-967-2

ISSN : $1254-5740$

Référence électronique

Jean Quétier, « Marx, éthicien anarchiste? », Les Cahiers philosophiques de Strasbourg [En ligne], 41 |

2017, mis en ligne le 03 décembre 2018, consulté le 14 novembre 2022. URL : http://

journals.openedition.org/cps/302 ; DOI : https://doi.org/10.4000/cps.302

\section{(c) (†) (2)}

Creative Commons - Attribution - Pas d'Utilisation Commerciale - Partage dans les Mêmes Conditions 4.0 International - CC BY-NC-SA 4.0

https://creativecommons.org/licenses/by-nc-sa/4.0/ 


\title{
Maximilien Rubel, Karl Marx. Essai de biographie intellectuelle, préface de Louis Janover, Paris: Klincksieck, coll. «Critique de la politique», 2016 (464 p.)
}

\author{
Marx, éthicien anarchiste?
}

Jean Quétier

Les éditions Klincksieck viennent de rééditer Karl Marx, essai de biographie intellectuelle de Maximilien Rubel, paru pour la première fois en 1957. L'ouvrage est issu de la thèse de doctorat de l'auteur, chercheur au CNRS disparu en 1996 et notamment célèbre pour son édition controversée des œuvres de Marx dans la Bibliothèque de la Pléiade ${ }^{1}$. L'importante synthèse du parcours théorique de Marx que l'on trouve dans le livre faisait en son temps partie des travaux universitaires pionniers sur la question. Foisonnant de citations, l'ouvrage constitue par la même occasion une plongée dans les textes de Marx. Il est évident que la recherche marxologique a considérablement progressé depuis, notamment grâce au remarquable travail éditorial mené depuis les années 1970 par l'équipe de la Marx-Engels Gesamtausgabe, l'édition critique des ouvres de Marx et Engels en langue d'écriture, et on peut être surpris du fait que les éditeurs de 2016 n'aient pas jugé utile de mentionner ce point qui rend l'entreprise de Maximilien Rubel - malgré ses mérites indéniables - inévitablement datée.

1 Sur ce point, nous renvoyons à l'article déjà ancien d'Eustache KouvÉLAKIs, "Marx encore! Questions d'édition de l'œuvre marxienne», in: Futur antérieur, n ${ }^{\circ}$ 30-31-32, Paris, 1995, et à la thèse pour le diplôme d'archiviste paléographe d'Aude Le Moullec-Rieu soutenue en 2015 à l'École nationale des chartes: Maximilien Rubel, éditeur de Marx dans la Bibliothèque de la Pléiade (1955-1968). 
On sera plus étonné encore de constater que la nouvelle édition reprend plusieurs erreurs factuelles qui ne peuvent être mises au compte des limites de la recherche de l'époque et qui reviennent trop fréquemment dans l'ouvrage pour qu'on les considère comme de simples coquilles. Ainsi en va-t-il de l'affirmation - tout sauf secondaire pour un auteur qui considère que l'inspiration de Marx est d'abord éthique - selon laquelle les thèses principales de l'éthique sociale de Marx se rattacheraient, entre autres, à "la philosophie stoïcienne $»^{2}$. Preuve à l'appui: sa tentative de «rattacher le matérialisme français et anglais à la philosophie de Démocrite et d'Épicure " ${ }^{3}$ dans La Sainte Famille... La confusion entre stoïcisme et épicurisme est patente et on pourrait la mettre sur le compte d'une pardonnable erreur d'inattention si elle n'était reprise explicitement comme une définition synthétique du matérialisme de Marx dans la préface de l'ouvrage 4 . De même en va-t-il pour un certain jeune hégélien dénommé "Ludwig Strauss", cité à quatre reprises et même répertorié dans l'index des noms ${ }^{5}$, dont on devine qu'il est le fruit d'une confusion entre David Friedrich Strauss et Ludwig Feuerbach...

La réédition est accompagnée d'une préface inédite de Louis Janover. Les premières lignes donnent le ton: il s'agit de revenir à Marx contre le marxisme. Rien d'étonnant quand on connaît le parcours de Rubel, proche du conseillisme, qui fut, avec Raymond Aron de l'autre côté de l'échiquier politique, un des premiers promoteurs de l'adjectif «marxien». On ne peut que partager les critiques formulées par Janover à l'égard du stalinisme et du marxisme dogmatique en général, mais l'auteur semble oublier que le marxisme est et a toujours été pluriel. La préface est malheureusement souvent approximative: le marxisme sert d'épouvantail mais Janover ne se confronte presque jamais aux textes eux-mêmes. Rédigée en 2016, elle conserve pourtant un arrière-goût de guerre froide: aucun auteur postérieur à la chute du mur de Berlin n'y est cité, alors même que la période contemporaine foisonne de travaux sur Marx. On regrettera que Janover se contente le plus souvent

2 M. Rubel, Karl Marx. Essai de biographie intellectuelle, p. 123.

3 Ibid., p. 122.

4 L. Janover "Marx tel qu'en lui-même", in: M. Rubel, Karl Marx. Essai de biographie intellectuelle, $\mathrm{p} . \mathrm{xx}$.

5 M. Rubel, Karl Marx. Essai de biographie intellectuelle, p. 385. 
d'affirmations péremptoires et, il faut bien le dire, injustifiées. Hors Rubel, il n'y aurait point de salut, et le Marx du XXI siècle, "tout en se présentant libéré du passé», ne vaudrait "guère mieux", selon Janover, "que les Marx qui se sont succédé sur la scène de l'histoire, quand le totalitarisme et le capitalisme se disputaient pour savoir où classer cet étrange empêcheur de penser en rond dans la constellation marxiste ${ }^{6}$.

Mais quel est donc le Marx que Rubel veut opposer au marxisme? C'est d'abord un Marx «éthicien». Mettant en avant dans chaque texte de Marx ce qu'il considère être des jugements de valeur ou des postulats normatifs, il entend briser l'image de froideur scientifique offerte par le marxisme. Pour Rubel, il est possible de repérer quelques grandes intuitions éthiques qui traversent l'œuvre de Marx depuis sa prime jeunesse - notamment l'idée selon laquelle la perfection individuelle est dépendante du bonheur de tous - et que ses travaux «sociologiques" auront pour vocation de consolider. L'importance que Rubel accorde à la dimension existentielle et même affective de l'engagement de Marx le conduit à opérer des rapprochements surprenants, notamment avec Kierkegaard, considérant que la critique que les deux auteurs adressent à Hegel est un élément qui leur est commun. Par ailleurs, le sens que Rubel donne à la notion même d'éthique est quelque peu imprécis (le prolétariat est un concept "éthique», le communisme est un concept "éthique», etc.) et l'on peine parfois à voir ce qui la distingue de la politique. La thèse de Rubel n'est pas sans risque: à de nombreuses reprises, en prétendant renvoyer les thèses de Marx à une position éthique, il semble en réalité les renvoyer à une position purement subjective voire arbitraire ou, selon ses propres mots, "affecti[ve] » ${ }^{7}$. En effet, comment comprendre autrement, par exemple, l'affirmation selon laquelle "Le Capital est sans doute un des livres les plus subjectifs $»^{8}$ ? Rubel voit ainsi dans la survaleur elle-même un concept dont la racine est d'abord éthique, dans la mesure où elle découle "d'un rapport social qui signifie en premier lieu l'écrasement du non-propriétaire par le propriétaire au moyen de l'État, l'aliénation économique et sociale»". Selon Rubel, on

6 L. JANover, «Marx tel qu'en lui-même», in: M. Rubel, Karl Marx. Essai de biographie intellectuelle, $\mathrm{p}$. L.

7 M. RubeL, Karl Marx. Essai de biographie intellectuelle, p. 295.

8 Ibid., p. 273.

9 Ibid., p. 39, n. 3. 
pourrait en trouver la preuve dans le fait que le terme Mehrwert apparaît sous la plume de Marx dès les articles de la Gazette rhénane sur les vols de bois en 1842, avant même donc que Marx se lance dans l'étude de l'économie politique. Mais n'est-ce pas là négliger l'importance centrale que Marx confère à la "critique des catégories économiques ${ }^{10}$ et sousentendre que l'élaboration d'une théorie de l'exploitation - à laquelle Marx a consacré au bas mot trente années de sa vie - relève tout au plus de la formalisation d'une intuition? N'est-ce pas confondre le mot et le concept, comme le faisaient déjà ceux qui, comme Anton Menger, reprochaient à Marx d'avoir purement et simplement repris son concept de survaleur au ricardien de gauche William Thompson ${ }^{11}$ ?

Le Marx de Rubel est également un Marx anarchiste. Il est vrai que, dès les années 1840, Marx cesse de voir dans l'État le vecteur de l'émancipation sociale et qu'il considère son dépérissement comme une nécessité pour le mouvement communiste. Cela suffit-il à faire de Marx un anarchiste? Selon Rubel, "sans que le mot soit jamais prononcé»" ${ }^{12}$, c'est bien le sens profond de la conception que l'on retrouve dans le Manuscrit de Kreuznach. Il est permis d'être moins catégorique: Marx a également insisté tout au long de sa vie sur l'importance stratégique de la conquête du pouvoir politique par la classe ouvrière, et la lutte qu'il a menée contre les bakouninistes a été âpre. Il est d'ailleurs très surprenant, au regard de la thèse audacieuse défendue par Rubel, que ce dernier se contente d'évoquer en une page ${ }^{13}$ les désaccords de fond qui ont opposé Marx aux anarchistes au sein de l'Internationale, conduisant à la scission du congrès de La Haye en 1872. On peut d'ailleurs considérer que la dictature révolutionnaire du prolétariat, forme prise par l'État lors de la "période de transformation révolutionnaire ${ }^{14}$ de la société capitaliste en société communiste ne constitue pas, en réalité, le seul

10 Lettre de Karl Marx à Ferdinand Lassalle du 22 février 1858, in: Karl Marx, Friedrich Engels, Correspondance, t. V, Paris: Éditions sociales, 1975, p. 143.

11 Sur ce point, $c f$. notamment Friedrich Engels, Karl Kautsky, JuristenSozialismus [1886], MEGA² I/31, p. 397 sqq., ou encore le récent article de Claude Morilhat, "William Thompson, l'inventeur du concept de survaleur?», in: La Pensée, n 379, Paris, 2014.

12 M. Rubel, Karl Marx. Essai de biographie intellectuelle, p. 49.

13 Ibid., p. 333.

14 K. MARX, Critique du programme de Gotha (1875), Paris: Éditions sociales, GEME, 2008, p. 73. 
point de désaccord entre Marx et les anarchistes, contrairement à ce que suggère Rubel. Ce que Marx reproche à «l'indifférence en matière de politique ${ }^{15}$, c'est aussi d'entraver toutes les conquêtes politiques et économiques possibles de la classe ouvrière (réduction du temps de travail, interdiction du travail des enfants...), de désorganiser le mouvement prolétarien lui-même et, en définitive, de faire le jeu de la bourgeoisie et des tenants de l'ordre établi. Contre la lecture proposée par Rubel, il paraît finalement plus raisonnable de voir en Marx, comme il le faisait lui-même, un communiste.

15 K. MARX, L'Indifferenza in materia politica (1872), MEGA² I/24, p. 105 sq. 